\title{
On Certain Class of Bazilevič Functions Associated with the Lemniscate of Bernoulli
}

\author{
Tamer M. Seoudy $\mathbb{D}^{1,2}$ and Amnah E. Shammaky ${ }^{3}$ \\ ${ }^{1}$ Department of Mathematics, Faculty of Science, Fayoum University, Fayoum 63514, Egypt \\ ${ }^{2}$ Department of Mathematics, Jamoum University College, Umm Al-Qura University, Makkah, Saudi Arabia \\ ${ }^{3}$ Department of Mathematics, Faculty of Science, Jazan University, Jazan, Saudi Arabia \\ Correspondence should be addressed to Tamer M. Seoudy; tms00@fayoum.edu.eg
}

Received 17 November 2020; Revised 1 December 2020; Accepted 3 December 2020; Published 12 December 2020

Academic Editor: Gangadharan MURUGUSUNDARAMOORTHY

Copyright (c) 2020 Tamer M. Seoudy and Amnah E. Shammaky. This is an open access article distributed under the Creative Commons Attribution License, which permits unrestricted use, distribution, and reproduction in any medium, provided the original work is properly cited.

Making use of the principle of subordination, we introduce a certain class of multivalently Bazilevic functions involving the Lemniscate of Bernoulli. Also, we obtain subordination properties, inclusion relationship, convolution result, coefficients estimate, and Fekete-Szegö problem for this class.

\section{Introduction}

Let $\mathscr{H}(\mathbb{U})$ be the class of analytic functions in the open unit disk

$$
\mathbb{U}=\{\zeta \in \mathbb{C}:|\zeta|<1\}
$$

and let $\mathscr{A}_{p}$ denote the subclass of $\mathscr{H}(\mathbb{U})$ consisting of functions of the form:

$$
f(\zeta)=\zeta^{p}+\sum_{k=p+1}^{\infty} a_{k} \zeta^{k}(p \in \mathbb{N}=\{1,2,3, \cdots\}) .
$$

We write $\mathscr{A}_{1}=\mathscr{A}$. For $f_{1}, f_{2} \in \mathscr{H}(\mathbb{U})$, we say that $f_{1}(\zeta)$ is subordinate to $f_{2}(\zeta)$, written symbolically, $f_{1} \prec f_{2}$ in $\mathbb{U}$ or $f_{1}$ $(\zeta) \prec f_{2}(\zeta)(\zeta \in \mathbb{U})$, if there exists a Schwarz function $\omega(\zeta)$, which (by definition) is analytic in $\mathbb{U}$ with $\omega(0)=0$ and $|\omega(\zeta)|<1(\zeta \in \mathbb{U})$ such that $f_{1}(\zeta)=f_{2}(\omega(\zeta))(\zeta \in \mathbb{U})$. Further more, if the function $f_{2}(\zeta)$ is univalent in $\mathbb{U}$, then we have the following equivalence (see $[1,2])$ :

$$
f_{1}(\zeta) \prec f_{2}(\zeta)(\zeta \in \mathbb{U}) \Leftrightarrow f_{1}(0)=f_{2}(0) \text { and } f_{1}(\mathbb{U}) \subset f_{2}(\mathbb{U})
$$

Let $\phi: \mathbb{C}^{2} \times \mathbb{U} \longrightarrow \mathbb{C}$ and $h(\zeta)$ be univalent in $\mathbb{U}$. If $g(\zeta)$ is analytic in $\mathbb{U}$ and satisfies the first order differential subordination:

$$
\phi\left(g(\zeta), \zeta g^{\prime}(\zeta) ; \zeta\right) \prec h(\zeta)
$$

then $g(\zeta)$ is a solution of the differential subordination (4). The univalent function $q(\zeta)$ is called a dominant of the solutions of the differential subordination (4) if $g(\zeta) \prec q(\zeta)$ for all $g(\zeta)$ satisfying (4). A univalent dominant $\tilde{q}$ that satisfies $\tilde{q} \prec q$ for all dominants of (4) is called the best dominant.

Sokól and Stankiewicz [3] introduced the class $\mathcal{S} \mathscr{L}^{*}$ consisting of analytic functions $f \in \mathscr{A}$ satisfying the following condition

$$
\left|\left[\frac{\zeta f^{\prime}(\zeta)}{f(\zeta)}\right]^{2}-1\right|<1
$$

which is equivalent to

$$
\frac{\zeta f^{\prime}(\zeta)}{f(\zeta)} \prec q(\zeta)=\sqrt{1+\zeta},
$$


where the function

$$
q(\zeta)=\sqrt{1+\zeta}(\zeta \in \mathbb{U})
$$

maps $\mathbb{U}$ onto the domain $\mathcal{O}=\left\{w \in \mathbb{C}: \mathfrak{R} w>0,\left|w^{2}-1\right|<1\right\}$, and its boundary $\partial \mathcal{O}$ is the right-half of the lemniscate of Bernoulli $\left(x^{2}+y^{2}\right)^{2}-2\left(x^{2}-y^{2}\right)=0$. Several geometric properties of $\delta \mathscr{L}^{*}$ were investigated done by many authors in $([4-7])$.

Now, we define a class $\mathscr{B}_{p}(\lambda, \alpha)$ of Bazilevic functions associated with lemniscate of Bernoullia by using the principle of differential subordination as follows.

Definition 1. A function $f \in \mathscr{A}_{p}$ is said to be the class $\mathscr{B}_{p}(\lambda$, $\alpha)$ if it satisfies the following subordination condition:

$$
(1-\lambda)\left(\frac{f(\zeta)}{\zeta^{p}}\right)^{\alpha}+\lambda \frac{\zeta f^{\prime}(\zeta)}{p f(\zeta)}\left(\frac{f(\zeta)}{\zeta^{p}}\right)^{\alpha} \prec \sqrt{1+\zeta}
$$

all the powers are principal values and throughout the paper unless otherwise mentioned the parameters $\lambda, \alpha$, and $p$ are constrained as $\lambda \in \mathbb{C}, \alpha>0, p \in \mathbb{N}$, and $\zeta \in \mathbb{U}$.

We note that

(1) $\mathscr{B}_{1}(\lambda, \alpha)=\mathscr{B}(\lambda, \alpha)=\left\{f \in \mathscr{A}:(1-\lambda)(f(\zeta) / \zeta)^{\alpha}+\right.$ $\left.\lambda\left(\zeta f^{\prime}(\zeta) / f(\zeta)\right)(f(\zeta) / \zeta)^{\alpha}<\sqrt{1+\zeta}\right\}$

(2) $\mathscr{B}_{p}(\lambda, 1)=\mathscr{B}_{p}(\lambda)=\left\{f \in \mathscr{A}_{p}:(1-\lambda)\left(f(\zeta) / \zeta^{p}\right)+\right.$ $\left.\lambda\left(f^{\prime}(\zeta) / p \zeta^{p-1}\right) \prec \sqrt{1+\zeta}\right\}$ and $\mathscr{B}_{1}(\lambda)=\mathscr{B}(\lambda)$

(3) $\mathscr{B}_{p}(1, \alpha)=\mathscr{B}_{p}(\alpha)=\left\{f \in \mathscr{A}_{p}:\left(\zeta f^{\prime}(\zeta) / p f(\zeta)\right)\right.$ $\left.\left(f(\zeta) / \zeta^{p}\right)^{\alpha}<\sqrt{1+\zeta}\right\}$ and $\mathscr{B}_{1}(\alpha)=\mathscr{B}(\alpha)$

(4) $\mathscr{B}_{p}(1,0)=\mathcal{S}_{p}^{*}=\left\{f \in \mathscr{A}_{p}:\left(\zeta f^{\prime}(\zeta) / p f(\zeta)\right) \prec\right.$ $\sqrt{1+\zeta}\}$ and $\delta \mathscr{L}_{1}^{*}=\delta \mathscr{L}^{*}$

In order to establish our main results, we need the following lemmas.

Lemma 2 [8]. Let the function $h$ be analytic and convex (univalent) in $\mathbb{U}$ with $h(0)=1$. Suppose also that the function $g(\zeta)$ given by

$$
g(\zeta)=1+c_{1} \zeta+c_{2} \zeta^{2}+\cdots
$$

is analytic in $\mathbb{U}$. If

$$
g(\zeta)+\frac{\zeta g^{\prime}(\zeta)}{\gamma} \prec h(\zeta)(\Re(\gamma) \geq 0 ; \gamma \neq 0 ; \zeta \in \mathbb{U})
$$

then

$$
g(\zeta) \prec q(\zeta)=\gamma \zeta^{-\gamma} \int_{0}^{\zeta} h(t) t^{\gamma-1} \quad d t \prec h(\zeta),
$$

and $q(\zeta)$ is the best dominant.
Lemma 3. [9]. For real or complex numbers $a, b, c(c \neq 0,-1$, $-2, \cdots)$ and $\zeta \in \mathbb{U}$,

$$
\begin{aligned}
& \int_{0}^{1} t^{b-1}(1-t)^{c-b-1}(1-t \zeta)^{-a} d t \\
& \quad=\frac{\Gamma(b) \Gamma(c-b)}{\Gamma(c)} F_{2}(a, b ; c ; \zeta) \quad(\Re(c)>\Re(b)>0), \\
& { }_{2} F_{1}(a, b ; c ; \zeta)=(1-\zeta)_{2}^{-a} F_{1}\left(a, c-b ; c ; \frac{\zeta}{\zeta-1}\right) .
\end{aligned}
$$

Lemma 4. [10]. Let $F$ be analytic and convex in $\mathbb{U}$. If $f, g \prec F$, then

$$
\lambda f+(1-\lambda) g \prec F(0 \leq \lambda \leq 1) .
$$

Lemma 5 [11]. Let $f(\zeta)=\sum_{k=1}^{\infty} a_{k} \zeta^{k}$ be analytic in $\mathbb{U}$ and $g(\zeta)=\sum_{k=1}^{\infty} b_{k} \zeta^{k}$ be analytic and convex in $\mathbb{U}$. If $f<g$, then

$$
\left|a_{k}\right|<\left|b_{1}\right|(k \in \mathbb{N}) .
$$

Lemma 6 [12]. Let $g(\zeta)=1+\sum_{k=1}^{\infty} c_{k} \zeta^{k} \in \mathscr{P}$, i.e., let $g$ be analytic in $\mathbb{U}$ and satisfy $\mathfrak{R}\{g(\zeta)\}>0$ for $\zeta \in \mathbb{U}$, then the following sharp estimate holds

$$
\left|c_{2}-v c_{1}^{2}\right| \leq 2 \max \{1,|2 v-1|\} \text { for all } v \in \mathbb{C} .
$$

The result is sharp for the functions given by

$$
g(\zeta)=\frac{1+\zeta^{2}}{1-\zeta^{2}} \text { or } g(\zeta)=\frac{1+\zeta}{1-\zeta}
$$

Lemma 7. [12]. If $g(\zeta)=1+\sum_{k=1}^{\infty} c_{k} \zeta^{k} \in \mathscr{P}$, then

$$
\left|c_{2}-v c_{1}^{2}\right| \leq\left\{\begin{array}{ccc}
-4 v+2 & \text { if } & v \leq 0, \\
2 & \text { if } & 0 \leq v \leq 1, \\
4 v-2 & \text { if } & v \geq 1,
\end{array}\right\}
$$

when $v<0$ or $v>1$, the equality holds if and only if $g(\zeta)$ $=(1+\zeta) /(1-\zeta)$ or one of its rotations. If $0<v<1$, then the equality holds if and only if $g(\zeta)=\left(1+\zeta^{2}\right) /\left(1-\zeta^{2}\right)$ or one of its rotations. If $v=0$, the equality holds if and only if

$$
g(\zeta)=\left(\frac{1+\lambda}{2}\right) \frac{1+\zeta}{1-\zeta}+\left(\frac{1-\lambda}{2}\right) \frac{1-\zeta}{1+\zeta}(0 \leq \lambda \leq 1)
$$

or one of its rotations. If $v=1$, the equality holds if and only if $g$ is the reciprocal of one of the functions such that equality holds in the case of $v=0$. 
Also, the above upper bound is sharp, and it can be improved as follows when $0<v<1$ :

$$
\begin{array}{r}
\left|c_{2}-v c_{1}^{2}\right|+v\left|c_{1}\right|^{2} \leq 2\left(0 \leq v \leq \frac{1}{2}\right), \\
\left|c_{2}-v c_{1}^{2}\right|+(1-v)\left|c_{1}\right|^{2} \leq 2\left(\frac{1}{2} \leq v \leq 1\right) .
\end{array}
$$

In the present paper, we obtain subordination properties, inclusion relationship, convolution result, coefficients estimate, and Fekete-Szegö inequalities for the class $\mathscr{B}_{p}(\lambda, \alpha)$.

\section{Main Results}

We begin by presenting our first subordination property given by Theorem 8 .

Theorem 8. If $f \in \mathscr{B}_{p}(\lambda, \alpha)$ with $\mathfrak{R}(\lambda)>0$, then

$$
\left(\frac{f(\zeta)}{\zeta^{p}}\right)^{\alpha} \prec Q(\zeta) \prec \sqrt{1+\zeta}
$$

where the function $Q(\zeta)$ given by

$$
Q(\zeta)=(1+\zeta){ }_{2}^{1 / 2} F_{1}\left(-\frac{1}{2}, 1 ; \frac{p \alpha}{\lambda}+1 ; \frac{\zeta}{1+\zeta}\right)
$$

is the best dominant.

Proof. Let $f \in \mathscr{B}_{p}(\lambda, \alpha)$ and suppose that

$$
g(\zeta)=\left(\frac{f(\zeta)}{\zeta^{p}}\right)^{\alpha} \quad(\zeta \in \mathbb{U})
$$

Then, the function $g(\zeta)$ is of the form (9), analytic in $\mathbb{U}$, and $g(0)=1$. By taking the derivatives in the both sides of (22), we get

$$
(1-\lambda)\left(\frac{f(\zeta)}{\zeta^{p}}\right)^{\alpha}+\lambda \frac{\zeta f^{\prime}(\zeta)}{p f(\zeta)}\left(\frac{f(\zeta)}{\zeta^{p}}\right)^{\alpha}=g(\zeta)+\frac{\lambda}{p \alpha} \zeta g^{\prime}(\zeta)
$$

Since $f \in \mathscr{B}_{p}(\lambda, \alpha)$, we have

$$
g(\zeta)+\frac{\lambda}{p \alpha} \zeta g^{\prime}(\zeta) \prec \sqrt{1+\zeta}
$$

Now, by using Lemma 2 for $\gamma=p \alpha / \lambda$, we deduce that

$$
\begin{aligned}
\left(\frac{f(\zeta)}{\zeta^{p}}\right)^{\alpha} & \prec Q(\zeta)=\frac{p \alpha}{\lambda} \zeta^{-(p \alpha / \lambda)} \int_{0}^{\zeta} t^{(p \alpha / \lambda)-1}(1+t)^{1 / 2} d t \\
& =\frac{p \alpha}{\lambda} \int_{0}^{1} u^{(p \alpha / \lambda)-1}(1+\zeta u)^{1 / 2} d u \\
& =(1+\zeta)^{1 / 2}{ }_{2} F_{1}\left(-\frac{1}{2}, 1 ; \frac{p \alpha}{\lambda}+1 ; \frac{\zeta}{1+\zeta}\right),
\end{aligned}
$$

where we have made a change of variables followed by the use of identities in Lemma 3 with $a=-1 / 2, b=p \alpha / \lambda n$, and $c=b+1$. This completes the proof of Theorem 8 .

For a function $f \in \mathscr{A}(p)$ given by (2), the generalized Bernardi-Libera-Livingston integral operator $F_{p, \mu}: \mathscr{A}(p)$ $\longrightarrow \mathscr{A}(p)$, with $\mu>-p$, is defined by (see [13-16])

$$
F_{p, \mu} f(\zeta)=\frac{\mu+p}{\zeta^{\mu}} \int_{0}^{\zeta} t^{\mu-1} f(t) d t(\mu>-p)
$$

It is easy to verify that for all $f \in \mathscr{A}(p)$, we have

$$
\zeta\left(F_{p, \mu} f(\zeta)\right)^{\prime}=(\mu+p) f(\zeta)-\mu F_{p, \mu} f(\zeta) .
$$

Theorem 9. If the function $f \in \mathscr{A}(p)$ satisfies the subordination condition

$$
(1-\lambda)\left(\frac{F_{p, \mu} f(\zeta)}{\zeta^{p}}\right)^{\alpha}+\lambda \frac{f(\zeta)}{F_{p, \mu} f(\zeta)}\left(\frac{F_{p, \mu} f(\zeta)}{\zeta^{p}}\right)^{\alpha} \prec \sqrt{1+\zeta}
$$

and $F_{p, \mu}$ is the integral operator defined by (26), then

$$
\left(\frac{F_{p, \mu} f(\zeta)}{\zeta^{p}}\right)^{\alpha} \prec K(\zeta) \prec \sqrt{1+\zeta}
$$

where the function $K$ given by

$$
K(\zeta)=(1+\zeta){ }_{2}^{1 / 2} F_{1}\left(-\frac{1}{2}, 1 ; \frac{\alpha(p+\mu)}{\lambda}+1 ; \frac{\zeta}{1+\zeta}\right)
$$

is the best dominant of (28).

Proof. Let

$$
g(\zeta)=\left(\frac{F_{p, \mu} f(\zeta)}{\zeta^{p}}\right)^{\alpha}(\zeta \in \mathbb{U})
$$

then $g$ is analytic in $\mathbb{U}$. Differentiating (31) with respect to $\zeta$ and using the identity (28) in the resulting relation, we get

$$
\begin{gathered}
(1-\lambda)\left(\frac{F_{p, \mu} f(\zeta)}{\zeta^{p}}\right)^{\alpha}+\lambda \frac{f(\zeta)}{F_{p, \mu} f(\zeta)}\left(\frac{F_{p, \mu} f(\zeta)}{\zeta^{p}}\right)^{\alpha} \\
=g(\zeta)+\frac{\lambda \zeta g^{\prime}(\zeta)}{\alpha(p+\mu)} \prec \sqrt{1+\zeta} .
\end{gathered}
$$

Employing the same technique that we used in the proof of Theorem 8, the remaining part of the theorem can be proved similarly.

Theorem 10. If $\lambda_{2} \geq \lambda_{1} \geq 0$, then

$$
\mathscr{B}_{p}\left(\lambda_{2}, \alpha\right) \subset \mathscr{B}_{p}\left(\lambda_{1}, \alpha\right) .
$$


Proof. Suppose that $f \in \mathscr{B}_{p}\left(\lambda_{2}, \alpha\right)$. We know that

$$
\left(1-\lambda_{2}\right)\left(\frac{f(\zeta)}{\zeta^{p}}\right)^{\alpha}+\lambda_{2} \frac{\zeta f^{\prime}(\zeta)}{p f(\zeta)}\left(\frac{f(\zeta)}{\zeta^{p}}\right)^{\alpha} \prec \sqrt{1+\zeta}
$$

Thus, the assertion of Theorem 10 holds for $\lambda_{2}=\lambda_{1} \geq 0$. If $\lambda_{2}>\lambda_{1} \geq 0$, by Theorem 8 and (34), we have

$$
\left(\frac{f(\zeta)}{\zeta^{p}}\right)^{\alpha} \prec \sqrt{1+\zeta}
$$

At the same time, we have

$$
\begin{aligned}
(1- & \left.\lambda_{1}\right)\left(\frac{f(\zeta)}{\zeta^{p}}\right)^{\alpha}+\lambda_{1} \frac{\zeta f^{\prime}(\zeta)}{p f(\zeta)}\left(\frac{f(\zeta)}{\zeta^{p}}\right)^{\alpha} \\
= & \left(1-\frac{\lambda_{1}}{\lambda_{2}}\right)\left(\frac{f(\zeta)}{\zeta^{p}}\right)^{\alpha}+\frac{\lambda_{1}}{\lambda_{2}}\left[\left(1-\lambda_{2}\right)\left(\frac{f(\zeta)}{\zeta^{p}}\right)^{\alpha}\right. \\
& \left.+\lambda_{2} \frac{\zeta f^{\prime}(\zeta)}{p f(\zeta)}\left(\frac{f(\zeta)}{\zeta^{p}}\right)^{\alpha}\right]
\end{aligned}
$$

Moreover, since $0 \leq \lambda_{1} / \lambda_{2}<1$, and the function $\sqrt{1+\zeta}$ $(\zeta \in \mathbb{U})$ is analytic and convex in $\mathbb{U}$.

Combining (34)-(36) and Lemma 4, we find that

$$
\left(1-\lambda_{1}\right)\left(\frac{f(\zeta)}{\zeta^{p}}\right)^{\alpha}+\lambda_{1} \frac{\zeta f^{\prime}(\zeta)}{p f(\zeta)}\left(\frac{f(\zeta)}{\zeta^{p}}\right)^{\alpha} \prec \sqrt{1+\zeta}
$$

that is $f \in \mathscr{B}_{p}\left(\lambda_{1}, \alpha\right)$, which implies that the assertion (33) of Theorem 10 holds.

Theorem 11. If $f \in \mathscr{A}_{p}$, then $f \in \mathscr{B}_{p}(\lambda, \alpha)$ if and only if

$$
\left(\frac{f(\zeta)}{\zeta^{p}}\right)^{\alpha} *\left[\frac{1-L \zeta+M \zeta^{2}}{(1-\zeta)^{2}}\right] \neq 0 \quad(\zeta \in \mathbb{U})
$$

where

$$
\begin{aligned}
L & =\left(1+\frac{\lambda}{\alpha p}\right) e^{-i \theta}\left(1+\sqrt{1+e^{i \theta}}\right)+2 \\
M & =e^{-i \theta}\left(1+\sqrt{1+e^{i \theta}}\right)+1
\end{aligned}
$$

Proof. For any function $f \in \mathscr{A}_{p}$, we can verify that

$$
\begin{gathered}
\left(\frac{f(\zeta)}{\zeta^{p}}\right)^{\alpha}=\left(\frac{f(\zeta)}{\zeta^{p}}\right)^{\alpha} * \frac{1}{1-\zeta}, \\
\frac{\zeta f^{\prime}(\zeta)}{p f(\zeta)}\left(\frac{f(\zeta)}{\zeta^{p}}\right)^{\alpha}=\left(\frac{f(\zeta)}{\zeta^{p}}\right)^{\alpha} * \frac{1-(1-1 / p \alpha) \zeta}{(1-\zeta)^{2}}
\end{gathered}
$$

First, in order to prove that (38) holds, we will write (8) by using the principle of subordination, that is,

$$
(1-\lambda)\left(\frac{f(\zeta)}{\zeta^{p}}\right)^{\alpha}+\lambda \frac{\zeta f^{\prime}(\zeta)}{p f(\zeta)}\left(\frac{f(\zeta)}{\zeta^{p}}\right)^{\alpha}=\sqrt{1+w(\zeta)}
$$

where $w(\zeta)$ is a Schwarz function, hence

$$
(1-\lambda)\left(\frac{f(\zeta)}{\zeta^{p}}\right)^{\alpha}+\lambda \frac{\zeta f^{\prime}(\zeta)}{p f(\zeta)}\left(\frac{f(\zeta)}{\zeta^{p}}\right)^{\alpha} \neq \sqrt{1+e^{i \theta}}
$$

for all $\zeta \in \mathbb{U}$ and $\theta \in 0,2 \pi$ ). From (40) and (41), the relation (43) may be written as

$$
\left(\frac{f(\zeta)}{\zeta^{p}}\right)^{\alpha} *\left[\frac{1-\sqrt{1+e^{i \theta}}-\left(1-(\lambda / \alpha p)-2 \sqrt{1+e^{i \theta}}\right) \zeta-\sqrt{1+e^{i \theta}} \zeta^{2}}{(1-\zeta)^{2}}\right] \neq 0
$$

which is equivalent to

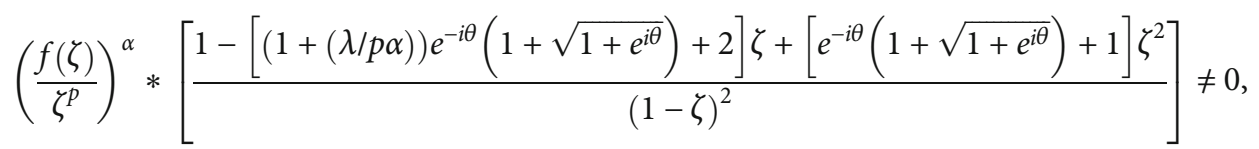


that is (38)

Reversely, suppose that $f \in \mathscr{A}_{p}$ satisfy the condition (38). Like it was previously shown, the assumption (38) is equivalent to (41), that is,

$$
\begin{aligned}
& (1-\lambda)\left(\frac{f(\zeta)}{\zeta^{p}}\right)^{\alpha}+\lambda \frac{\zeta f^{\prime}(\zeta)}{p f(\zeta)}\left(\frac{f(\zeta)}{\zeta^{p}}\right)^{\alpha} \\
& \neq \sqrt{1+e^{i \theta}} \quad(\zeta \in \mathbb{U}) .
\end{aligned}
$$

Denoting

$$
\begin{aligned}
\varphi(\zeta) & =(1-\lambda)\left(\frac{f(\zeta)}{\zeta^{p}}\right)^{\alpha}+\lambda \frac{\zeta f^{\prime}(\zeta)}{p f(\zeta)}\left(\frac{f(\zeta)}{\zeta^{p}}\right)^{\alpha} \text { and } \psi(\zeta) \\
& =\sqrt{1+\zeta}
\end{aligned}
$$

the relation (46) could be written as $\varphi(\mathbb{U}) \cap \psi(\partial \mathbb{U})=\varnothing$. Therefore, the simply connected domain $\varphi(\mathbb{U})$ is included in a connected component of $\mathbb{C} \backslash \psi(\partial \mathbb{U})$. From this fact, using that $\varphi(0)=\psi(0)=1$ together with the univalence of the function $\psi$, it follows that $\varphi(\zeta) \prec \psi(\zeta)$, that is $f \in \mathscr{B}_{p}$ $(\lambda, \alpha)$.

Theorem 12. If $f(\zeta)$ given by (2) belongs to $\mathscr{B}_{p}(\lambda, \alpha)$, then

$$
\left|a_{p+1}\right| \leq \frac{p}{2|p \alpha+\lambda|}
$$

Proof. Combining (2) and (8), we obtain

$$
\begin{aligned}
(1 & -\lambda)\left(\frac{f(\zeta)}{\zeta^{p}}\right)^{\alpha}+\lambda \frac{\zeta f^{\prime}(\zeta)}{p f(\zeta)}\left(\frac{f(\zeta)}{\zeta^{p}}\right)^{\alpha} \\
& =1+\left(\frac{p \alpha+\lambda}{p}\right) a_{p+1} \zeta+\cdots \cdot \prec \sqrt{1+\zeta} \\
& =1+\frac{1}{2} \zeta-\frac{1}{8} \zeta^{2}+\cdots .
\end{aligned}
$$

An application of Lemma 5 to (49) yields

$$
\left|\left(\frac{p \alpha+\lambda}{p}\right) a_{p+1}\right|<\frac{1}{2}
$$

Thus, from (50), we easily obtain (48) asserted by Theorem 12.

\section{Fekete-Szegö Problem}

Many authors have considered the Fekete-Szegö problem for many subclasses of analytic functions (see, for instance, [17$21])$. In this section, we evaluate the Fekete-Szegö inequalities for the class $\mathscr{B}_{p}(\lambda, \alpha)$.
Theorem 13. If $f$ given by (2) belongs to the class $\mathscr{B}_{p}(\lambda, \alpha)$, then

$$
\begin{aligned}
\left|a_{p+2}-\mu a_{p+1}^{2}\right| \leq & \frac{p}{2(\alpha p+2 \lambda)} \max \left\{1 ; \frac{1}{4} \mid 1\right. \\
& \left.+\frac{p(\alpha p+2 \lambda)(\alpha-1+2 \mu)}{(\alpha p+\lambda)^{2}} \mid\right\} .
\end{aligned}
$$

The result is sharp.

Proof. If $f \in \mathscr{B}_{p}(\lambda, \alpha)$, then there is a Schwarz function $\omega$ in $\mathbb{U}$ such that

$$
(1-\lambda)\left(\frac{f(\zeta)}{\zeta^{p}}\right)^{\alpha}+\lambda \frac{\zeta f^{\prime}(\zeta)}{p f(\zeta)}\left(\frac{f(\zeta)}{\zeta^{p}}\right)^{\alpha}=\phi(\omega(\zeta))
$$

where $\phi(\zeta)=\sqrt{1+\zeta}$. Define the function $g(\zeta)$ by

$$
g(\zeta)=\frac{1+\omega(\zeta)}{1-\omega(\zeta)}=1+c_{1} \zeta+c_{2} \zeta^{2}+\cdots
$$

Since $\omega(\zeta)$ is a Schwarz function, we see that $g \in \mathscr{P}$ with $g(0)=1$. Therefore,

$$
\begin{aligned}
\phi(\omega(\zeta)) & =\phi\left(\frac{g(\zeta)-1}{g(\zeta)+1}\right)=\sqrt{\frac{2 g(\zeta)}{g(\zeta)+1}} \\
& =1+\frac{1}{4} c_{1} \zeta+\left(\frac{1}{4} c_{2}-\frac{5}{32} c_{1}^{2}\right) \zeta^{2}+. .
\end{aligned}
$$

Now by substituting (54) in (52), we have

$$
\begin{aligned}
& (1-\lambda)\left(\frac{f(\zeta)}{\zeta^{p}}\right)^{\alpha}+\lambda \frac{\zeta f^{\prime}(\zeta)}{p f(\zeta)}\left(\frac{f(\zeta)}{\zeta^{p}}\right)^{\alpha} \\
& =1+\frac{1}{4} c_{1} \zeta+\left(\frac{1}{4} c_{2}-\frac{5}{32} c_{1}^{2}\right) \zeta^{2}+. \cdots
\end{aligned}
$$

Equating the coefficients of $\zeta$ and $\zeta^{2}$, we obtain

$$
\begin{aligned}
& a_{p+1}=\frac{p}{4(\alpha p+\lambda)} c_{1}, \\
& a_{p+2}=\frac{p}{4(\alpha p+2 \lambda)} c_{2}-\frac{p}{32}\left(\frac{5}{(\alpha p+2 \lambda)}+\frac{p(\alpha-1)}{(\alpha p+\lambda)^{2}}\right) c_{1}^{2} .
\end{aligned}
$$

Therefore,

$$
a_{p+2}-\mu a_{p+1}^{2}=\frac{p}{4(\alpha p+2 \lambda)}\left\{c_{2}-v c_{1}^{2}\right\}
$$


where

$$
v=\frac{1}{8}\left[5+\frac{p(\alpha p+2 \lambda)(\alpha-1+2 \mu)}{(\alpha p+\lambda)^{2}}\right] .
$$

Our result now follows by an application of Lemma 6 . The result is sharp for the functions

$$
\begin{aligned}
& (1-\lambda)\left(\frac{f(\zeta)}{\zeta^{p}}\right)^{\alpha}+\lambda \frac{\zeta f^{\prime}(\zeta)}{p f(\zeta)}\left(\frac{f(\zeta)}{\zeta^{p}}\right)^{\alpha}=\phi\left(\zeta^{2}\right) \\
& (1-\lambda)\left(\frac{f(\zeta)}{\zeta^{p}}\right)^{\alpha}+\lambda \frac{\zeta f^{\prime}(\zeta)}{p f(\zeta)}\left(\frac{f(\zeta)}{\zeta^{p}}\right)^{\alpha}=\phi(\zeta) .
\end{aligned}
$$

This completes the proof of Theorem 13.

Putting $\lambda=1$ and $\alpha=0$ in Theorem 13, we obtain the following corollary.

Corollary 14. If $f$ given by (2) belongs to the class $\mathscr{B}_{p}(\alpha)$, then

$$
\begin{aligned}
\left|a_{p+2}-\mu a_{p+1}^{2}\right| \leq & \frac{p}{2(\alpha p+2)} \max \left\{1 ; \frac{1}{4} \mid 1\right. \\
& \left.+\frac{p(\alpha p+2)(\alpha-1+2 \mu)}{(\alpha p+1)^{2}} \mid\right\} .
\end{aligned}
$$

The result is sharp.
Putting $\lambda=1$ and $\alpha=0$ in Theorem 13, we obtain the following corollary.

Corollary 15. If $f$ given by (2) belongs to the class $\mathcal{S L}_{p}^{*}$, then

$$
\left|a_{p+2}-\mu a_{p+1}^{2}\right| \leq \frac{p}{4} \max \left\{1 ; \frac{|1+2 p(2 \mu-1)|}{4}\right\} .
$$

The result is sharp.

Putting $p=\lambda=1$ and $\alpha=0$ in Theorem 13, we obtain the following corollary.

Corollary 16. If $f$ given by (2) (with $p=1)$ belongs to the class $\delta \mathscr{L}^{*}$, then

$$
\left|a_{3}-\mu a_{2}^{2}\right| \leq \frac{1}{4} \max \left\{1 ; \frac{|4 \mu-1|}{4}\right\} .
$$

The result is sharp.

Applying Lemma 7 to (57) and (58), we obtain the following theorem.

Theorem 17. Let

$$
\begin{aligned}
& \sigma_{1}=\frac{p(\alpha p+2 \lambda)(1-\alpha)-5(\alpha p+\lambda)^{2}}{2 p(\alpha p+2 \lambda)}, \\
& \sigma_{2}=\frac{p(\alpha p+2 \lambda)(1-\alpha)+3(\alpha p+\lambda)^{2}}{2 p(\alpha p+2 \lambda)}, \\
& \sigma_{3}=\frac{p(\alpha p+2 \lambda)(1-\alpha)-(\alpha p+\lambda)^{2}}{2 p(\alpha p+2 \lambda)} .
\end{aligned}
$$

If $f$ given by (2) belongs to the class $\mathscr{B}_{p}(\lambda, \alpha)$, then

$$
\left|a_{p+2}-\mu a_{p+1}^{2}\right| \leq \begin{cases}-\frac{p}{8(\alpha p+2 \lambda)}\left[1+\frac{p(\alpha p+2 \lambda)(\alpha-1+2 \mu)}{(\alpha p+\lambda)^{2}}\right] & \left(\mu \leq \sigma_{1}\right), \\ \frac{p}{2(\alpha p+2 \lambda)} & \left(\sigma_{1} \leq \mu \leq \sigma_{2}\right), \\ \frac{p}{8(\alpha p+2 \lambda)}\left[1+\frac{p(\alpha p+2 \lambda)(\alpha-1+2 \mu)}{(\alpha p+\lambda)^{2}}\right] & \left(\mu \geq \sigma_{2}\right) .\end{cases}
$$

Further, if $\sigma_{1} \leq \mu \leq \sigma_{3}$, then

$$
\begin{aligned}
& \left|a_{p+2}-\mu a_{p+1}^{2}\right|+\frac{1}{2}\left[\frac{5(\alpha p+\lambda)^{2}}{p(\alpha p+2 \lambda)}+\alpha-1+2 \mu\right]\left|a_{p+1}\right|^{2} \\
& \quad \leq \frac{p}{2(\alpha p+2 \lambda)} .
\end{aligned}
$$

If $\sigma_{3} \leq \mu \leq \sigma_{2}$, then

$$
\begin{aligned}
& \left|a_{p+2}-\mu a_{p+1}^{2}\right|+\frac{1}{2}\left[\frac{3(\alpha p+\lambda)^{2}}{p(\alpha p+2 \lambda)}-\alpha+1-2 \mu\right]\left|a_{p+1}\right|^{2} \\
& \quad \leq \frac{p}{2(\alpha p+2 \lambda)} .
\end{aligned}
$$



result.

Putting $\lambda=1$ in Theorem 17, we obtain the following

Corollary 18. Let

$$
\begin{aligned}
& \delta_{2}=\frac{p(\alpha p+2)(1-\alpha)+3(\alpha p+1)^{2}}{2 p(\alpha p+2)}, \\
& \delta_{3}=\frac{p(\alpha p+2)(1-\alpha)-(\alpha p+1)^{2}}{2 p(\alpha p+2)} .
\end{aligned}
$$

$$
\delta_{1}=\frac{p(\alpha p+2)(1-\alpha)-5(\alpha p+1)^{2}}{2 p(\alpha p+2)},
$$

If $f$ given by (2) belongs to the class $\mathscr{B}_{p}(\alpha)$, then

$$
\left|a_{p+2}-\mu a_{p+1}^{2}\right| \leq \begin{cases}-\frac{p}{8(\alpha p+2)}\left[1+\frac{p(\alpha p+2)(\alpha-1+2 \mu)}{(\alpha p+1)^{2}}\right] & \left(\mu \leq \delta_{1}\right), \\ \frac{p}{2(\alpha p+2)} & \left(\delta_{1} \leq \mu \leq \delta_{2}\right), \\ \frac{p}{8(\alpha p+2)}\left[1+\frac{p(\alpha p+2)(\alpha-1+2 \mu)}{(\alpha p+1)^{2}}\right] & \left(\mu \geq \delta_{2}\right) .\end{cases}
$$

Further, if $\delta_{1} \leq \mu \leq \delta_{3}$, then

$$
\begin{aligned}
& \left|a_{p+2}-\mu a_{p+1}^{2}\right|+\frac{1}{2}\left[\frac{5(\alpha p+1)^{2}}{p(\alpha p+2)}+\alpha-1+2 \mu\right]\left|a_{p+1}\right|^{2} \\
& \quad \leq \frac{p}{2(\alpha p+2)} .
\end{aligned}
$$

If $\delta_{3} \leq \mu \leq \delta_{2}$, then

$$
\begin{aligned}
& \left|a_{p+2}-\mu a_{p+1}^{2}\right|+\frac{1}{2}\left[\frac{3(\alpha p+1)^{2}}{p(\alpha p+2)}-\alpha+1-2 \mu\right]\left|a_{p+1}\right|^{2} \\
& \quad \leq \frac{p}{2(\alpha p+2)} .
\end{aligned}
$$

Putting $\lambda=1$ and $\alpha=0$ in Theorem 17, we obtain the following result for the subclass $\mathcal{S}_{\mathscr{L}_{p}^{*}}$.

Corollary 19. If $f$ given by (2) belongs to the class $\delta \mathscr{L}_{p}^{*}$, then

$$
\left|a_{p+2}-\mu a_{p+1}^{2}\right| \leq \begin{cases}-\frac{p[1+2 p(2 \mu-1)]}{16} & \left(\mu \leq \frac{2 p-5}{4 p}\right) \\ \frac{p}{4} & \left(\frac{2 p-5}{4 p} \leq \mu \leq \frac{2 p+3}{4 p}\right) \\ \frac{p[1+2 p(2 \mu-1)]}{16} & \left(\mu \geq \frac{2 p+3}{4 p}\right)\end{cases}
$$

Further, if $((2 p-5) / 4 p) \leq \mu \leq((2 p-1) / 4 p)$, then

$$
\begin{aligned}
& \left|a_{p+2}-\mu a_{p+1}^{2}\right|+\frac{1}{4}\left(\frac{5}{p}-2+4 \mu\right)\left|a_{p+1}\right|^{2} \leq \frac{p}{4} . \\
& \text { If }((2 p-1) / 4 p) \leq \mu \leq((2 p+3) / 4 p) \text {, then } \\
& \left|a_{p+2}-\mu a_{p+1}^{2}\right|+\frac{1}{4}\left(\frac{3}{p}+2-4 \mu\right)\left|a_{p+1}\right|^{2} \leq \frac{p}{4} .
\end{aligned}
$$

Putting $\lambda=p=1$ and $\alpha=0$ in Theorem 17, we obtain the following result obtained by ([18], Theorem 2.1).

Corollary 20. ([18], Theorem 2.1). If $f$ given by (2) (with $p=1$ ) belongs to the class $\mathcal{S} \mathscr{L}^{*}$, then

$$
\left|a_{3}-\mu a_{2}^{2}\right| \leq \begin{cases}-\frac{1}{16}(4 \mu-1) & \left(\mu \leq-\frac{3}{4}\right) \\ \frac{1}{4} & \left(-\frac{3}{4} \leq \mu \leq \frac{5}{4}\right) \\ \frac{1}{16}(4 \mu-1) & \left(\mu \geq \frac{5}{4}\right)\end{cases}
$$

Further, if $-(3 / 4) \leq \mu \leq 1 / 4$, then

$$
\left|a_{3}-\mu a_{2}^{2}\right|+\frac{1}{4}(3+4 \mu)\left|a_{2}\right|^{2} \leq \frac{1}{4} .
$$


If $1 / 4 \leq \mu \leq 5 / 4$, then

$$
\left|a_{3}-\mu a_{2}^{2}\right|+\frac{1}{4}(5-4 \mu)\left|a_{2}\right|^{2} \leq \frac{1}{4} .
$$

\section{Data Availability}

No data were used to support this study.

\section{Ethical Approval}

This article does not contain any studies with human participants or animals performed by any of the authors.

\section{Conflicts of Interest}

The authors declare that they have no competing interests.

\section{Authors' Contributions}

All authors contributed equally to the writing of this paper. All authors read and approved the final manuscript.

\section{References}

[1] T. Bulboaca, Differential Subordinations and Superordinations, Recent Results, House of Scientific Book Publ., Cluj-Napoca, 2005.

[2] S. S. Miller and P. T. Mocanu, "Differential subordination: theory and applications," in Series in Pure and Applied Mathematics, vol. 225, Marcel Dekker, New York, 2000.

[3] J. Sokól and J. Stankiewicz, "Radius of convexity of some subclasses of strongly starlike functions," Folia Scient. Univ. Tech. Resoviensis, Mat., vol. 19, pp. 101-105, 1996.

[4] R. M. Ali, N. E. Cho, V. Ravichandran, and S. S. Kumar, "Differential subordination for functions associated with the lemniscate of Bernoulli," Taiwanese Journal of Mathematics, vol. 16, no. 3, pp. 1017-1026, 2012.

[5] S. A. Halim and R. Omar, "Applications of certain functions associated with lemniscate Bernoulli," Journal of the Indonesian Mathematical Society, vol. 18, no. 2, pp. 93-99, 2012.

[6] J. Sokól, "Coefficient estimates in a class of strongly starlike functions," Kyungpook National University, vol. 49, no. 2, pp. 349-353, 2009.

[7] J. Sokól, "Radius problems in the class $\delta \mathscr{L}^{*}$," Applied Mathematics and Computation, vol. 214, no. 2, pp. 569-573, 2009.

[8] D. J. Hallenbeck and S. Ruscheweyh, "Subordination by convex functions," Proceedings of the American Mathematical Society, vol. 52, no. 1, pp. 191-195, 1975.

[9] E. T. Whittaker and G. N. Watson, A Course of Modern Analysis: An Introduction to the General Theory of Infinite Processes and of Analytic Functions; with an Account of the Principal Transcendental Functions, Cambridge University Press, Cambridge, 1927.

[10] M.-S. Liu, "On certain subclass of analytic functions," J. South China Normal Univ., vol. 4, pp. 15-20, 2002.

[11] W. Rogosinski, "On the coefficients of subordinate functions," Proceedings of the London Mathematical Society, vol. s2-48, no. 1, pp. 48-82, 1945.

[12] W. Ma and D. A. Minda, "Unified treatment of some special classes of univalent functions," Proceedings of the Conference on Complex Analysis, Tianjin, China, 19-23 June 1992, Z. Li, F. Ren, L. Yang, and S. Zhang, Eds., , pp. 157-169, Int. Press, Cambridge, MA, USA, 1994.

[13] S. D. Bernardi, "Convex and starlike univalent functions," Transactions of the American Mathematical Society, vol. 135, pp. 429-446, 1969.

[14] J. H. Choi, M. Saigo, and H. M. Srivastava, "Some inclusion properties of a certain family of integral operators," Journal of Mathematical Analysis and Applications, vol. 276, no. 1, pp. 432-445, 2002.

[15] R. J. Libera, "Some radius of convexity problems," Duke Mathematical Journal, vol. 31, no. 1, pp. 143-158, 1964.

[16] H. Saitoh, "On certain class of mulivalent functions," Mathematica Japonica, vol. 37, pp. 871-875, 1992.

[17] V. Ravichandran, A. Gangadharan, and M. Darus, "FeketeSzegö inequality for certain class of Bazilevic functions," Far East Journal of Mathematical Sciences, vol. 15, pp. 171-180, 2004.

[18] M. Raza and S. N. Malik, "Upper bound of the third Hankel determinant for a class of analytic functions related with lemniscate of Bernoulli," Journal of Inequalities and Applications, vol. 2013, no. 1, Article ID 412, 2013.

[19] T. M. Seoudy, "Fekete-Sezgö problems for certain class of nonBazilevic functions involving the Dziok-Srivastava operator," Romai J., vol. 10, no. 1, pp. 175-186, 2014.

[20] T. M. Seoudy and M. K. Aouf, "Coefficient estimates of new classes of q-starlike and q-convex functions of complex order," Journal of Mathematical Inequalities, vol. 10, no. 1, pp. 135145, 2007.

[21] T. N. Shanmugam, S. Sivassubramanian, and M. Darus, "Fekete-Szegö inequality for certain class of Bazilevic functions," Int. Math., vol. 34, pp. 283-290, 2006. 